\title{
Is Exclusive Breastfeeding Dangerous?
}

Share this:

Marsha Walker, RN, IBCLC, RLCa

Social media has been alight with descriptions of exclusive breastfeeding being dangerous, resulting in significant and severe negative outcomes in infants whose mothers wished to breastfeed. This backlash has been led by a campaign that uses inflammatory anecdotes and misleading and inaccurate interpretation of research to bolster its assault on breastfeeding. However, poor breastfeeding outcomes can and do happen. The narratives identify areas where clinicians can improve their delivery of care. A closer look at risk factors and interventions may help reduce the risk of poor outcomes and increase the likelihood of mothers meeting their breastfeeding goals.

Keywords: exclusive breastfeeding; negative outcomes; social media; lactation management

The Internet and social media have been rife with stories describing harmful outcomes in breastfeeding infants. Headlines have declared that exclusive breastfeeding has caused attention deficit disorder, hypoglycemia, hyperbilirubinemia, hypernatremia, dehydration, brain damage, and death in infants. The Baby-Friendly Hospital Initiative and lactation care providers have been blamed for serious complications in exclusively breastfed newborns because they promote breastfeeding and promulgate "dangerous" breastfeeding guidelines'.

While poor breastfeeding outcomes can and do occur, some social media postings and campaigns use fear, inflammatory anecdotes, misleading and inaccurate interpretation of research studies, and incorrect lactation-management guidelines that serve to undermine and confuse both mothers and clinicians regarding the safety and importance of exclusive breastfeeding. Such egregious dialog has even led the American College of Obstetricians and Gynecologists to issue alerts to its members regarding the sensationalized headlines with accusations that babies have been starved, experienced brain damage, and have been refused supplementary nutrition when needed (American College of Obstetricians and Gynecologists, 2018a, 2018b).

A number of social media platforms contain stories from mothers describing their struggles with breastfeeding. While the stories cannot be verified and many important details are lacking, the narratives identify areas where clinicians can improve their delivery of care (KendallTackett, 2018) and possess some commonalities (Box 1). This may provide clinicians with insights on how to enhance their practice and prevent poor breastfeeding outcomes. Exclusive breastfeeding is not dangerous, but

a. Marshalact@gmail.com responsible clinicians can view this as an opportunity to recognize that poor breastfeeding outcomes can happen, explore their etiology, and institute interventions that reduce and rectify them.

\section{Implications for Practice}

\section{Inconsolable Crying}

Inconsolable crying is not normal and differs from fussiness and "colic." A baby that feeds for hours at the breast and remains unsatisfied, engages in inconsolable crying, and cries when put down immediately after a feeding may indicate little to no colostrum/milk transfer. This situation requires a clinical evaluation of the feeding, which includes verification of both swallowing and colostrum/milk transfer. A pre- and post-feed weight can be done to assure that the infant is ingesting colostrum/ milk. Mothers experiencing this situation should be advised to seek immediate help from a suitable clinician who can perform the weighing and has the ability to assure or remedy milk transfer issues.

One social media site incorrectly labels this pattern as cluster feeding. Cluster feeding is a normal pattern of frequent feeds, usually in the late afternoon and early evening, not unremitting feeding and crying. Just because an infant is correctly latched to the breast does not guarantee milk transfer. Mothers can be taught the signs of infant swallowing (Box 2) so that they acquire both knowledge and skills to determine if their infant is feeding efficiently. Mothers should be able to tell if their baby is swallowing prior to discharge from the hospital. If mothers and clinicians are uncertain if the infant is swallowing, a stethoscope can be placed laterally on the baby's throat, called cervical auscultation, to pick up more subtle swallow sounds (Vice, Heinz, Giuriati, 
Box 1. Commonalities Among Mother-Reported Narratives on Social Media

Mothers describe inconsolable crying.

Infant feeding at breast nonstop for hours at a time.

Baby is unsatisfied following breastfeeding.

Healthcare provider stating that there is "correct latch."

No healthcare providers verify actual infant swallowing or colostrum/milk transfer.

Mother has sore/macerated nipples upon hospital discharge.

Guidelines for breastfeeding, expressing, supplementing guidelines are inappropriate (or misunderstood).

Hospitals have lack of written feeding plans for in-hospital and postdischarge.

Many are first-time mothers.
Hood, \& Bosma, 1990). If clinicians are still concerned regarding milk transfer, a pre- and post-feed weight can be done.

\section{Sore Nipples}

Sore nipples represent not only a frequent cause of breastfeeding abandonment but also an indicator of potentially problematic positioning, latch, suck, and/or milk intake. Sore nipples should be promptly addressed whether occurring in-hospital or postdischarge.

\section{Milk Production and Removal}

Inappropriate (or misunderstood) breastfeeding, expressing, and supplementing guidelines were commonly mentioned in a number of stories, especially surrounding mother-described breastfeeding problems. Mothers in these narratives, as well as those administering social media campaigns, attributed most problems to insufficient milk, not recognizing or acknowledging that many mothers may have sufficient colostrum and milk, but their infant may be unable to adequately remove it. A study of 280 new mothers found that suboptimal infant breastfeeding behaviors in newborns were common, with $49 \%$ of infants demonstrating suboptimal feeding patterns during the first 24 hours, $22 \%$ on day 3 , and $14 \%$ on day 7 .

Breastfeeding difficulties were significantly associated with first-time mothers, cesarean section, flat or inverted nipples, compromised infant status, supplementation with formula during the first 48 hours, pacifier use, maternal obesity, and low birth weight infants (Dewey, Nommsen-Rivers, Heinig, \& Cohen, 2003). This describes a cohort of mothers and infants requiring very close monitoring both in-hospital and postdischarge. For example, it is known that mothers with a body mass index $(\mathrm{BMI})>30$ experience delayed onset of lactation (Preusting, Brumley, Odibo, Spatz, \& Louis, 2017) and abandon breastfeeding earlier (Donath \& Amir, 2008) compared with women with a BMI <30. Yet, many healthcare providers do not believe that there is a difference in success rates between higher-BMI mothers and mothers with a BMI $<30$. Some have stated that mothers with a BMI $>30$ are given the same advice about breastfeeding as mothers with BMI <30 (Rasmussen, Lee, Ledkovsky, \& Kjolhede, 2006); however, recent research suggests that this is not the case (Kair \& Colaizy, 2016).

Fussy newborns, sleepy newborns, and nonlatching newborns all require specialized management guidelines and enhanced monitoring. Breastfeeding guidelines need to be very specific (Appendix A) and written down to avoid hypoglycemia, hyperbilirubinemia, 
dehydration, and other poor outcomes as described in social media stories. Some hospitals use whiteboards in postpartum rooms to write out each day's breastfeeding instructions.

\section{Insufficient Milk Versus Delayed Lactogenesis II}

There is also a difference between insufficient milk and delayed lactogenesis II, something social media campaigns and mother narratives fail to discern. Delayed onset of lactation has been defined as happening at $>72$ hours postpartum and is associated with first-time mothers, cesarean section, maternal BMI $>30$, certain labor medications, and infant status at birth (Dewey et al., 2003). Mothers with insulin-dependent diabetes and gestational diabetes can experience a 15- to 28-hour delay in lactogenesis II (Arthur, Kent, \& Hartmann, 1994; Bitman et al., 1989; Hartmann \& Cregan, 2001; Miyake, Tahara, Koike, \& Tanizawa, 1989; Murtaugh, Ferris, Capacchione, \& Reece, 1998; Ostrom \& Ferris, 1993), resulting in a prolonged colostral phase of lactation. For each 1-unit $\left(1 \mathrm{~kg} / \mathrm{m}^{2}\right)$ increase in prepregnant BMI, a 0.5-hour delay in the onset of lactogenesis II has been calculated (Hilson, Rasmussen, \& Kjolhede, 2004). The difference in onset of copious milk production can be up to 10 hours later in a mother with a BMI of 40 compared to a mother with a BMI of 20. Given the high probability for delayed onset of lactation in the group of mothers with one or more known risk factors, it is necessary to monitor these infants very closely and provide discharge instructions (Box 3) that account for a delay in copious milk availability.

\section{Hypoglycemia}

While relatively low concentrations of glucose are seen in all mammalian newborns, this is usually transient, asymptomatic, and part of a number of normal adaptations to postnatal life. However, occasional reports of full-term breastfeeding infants being readmitted for late-onset hypoglycemia persist (Seske, Merhar, \& Haberman, 2015). This can be worrisome as the newborn brain relies primarily on glucose and to a lesser extent other substrates to fuel its activity. Severe or prolonged hypoglycemia with clinical signs can result in neurologic injury.

\section{Box 3. Sample Discharge Instructions for Mothers With Known Risk for Delayed Lactogenesis II}

Breastfeed your infant 10 to 12 times each 24 hours until your milk comes in and baby is gaining weight well.

Use breast compressions with each suck to help transfer more colostrum.

Make sure you know how to tell if baby is swallowing.

If your baby does not latch or swallow well, hand express colostrum for each feeding. If you cannot express enough colostrum, ask the hospital to provide you with donor human milk or seek donor human milk from a human milk bank.

Feed your baby $2-10 \mathrm{ml} /$ feed (1-2 teaspoons) during the first 24 hours, $5-15 \mathrm{ml} /$ feed from 24 to 48 hours (1-3 teaspoons), $15-30 \mathrm{ml} /$ feed from 48 to 72 hours ( 3 teaspoons to 1 ounce or so), and $30-60 \mathrm{ml} /$ feed (1-2 ounces) from 72 to 96 hours.

Work with a lactation consultant who can weigh your baby at least once per day, and if necessary, perform pre- and post-feed weights to check baby's intake at a feeding.

Keep a diaper log of wet and dirty diapers. By day 3 or 4 , there should be three to four wet diapers and three to four dirty diapers. There should be no red crystals in a wet diaper after day 3 .

If baby's weight gain is not good, if baby becomes jaundiced, if baby cries constantly, if pre- and post-feed weights indicate little colostrum/milk transfer, if you cannot express enough colostrum, if no donor human milk is available, then a protein hydrolysate formula (hypoallergenic) may need to be temporarily used. 
There is a lack of consensus regarding the definition of hypoglycemia, when and who to treat, and how to treat infants during the early days of life (Adamkin \& Polin, 2016). Guidance documents from three organizations are frequently used to direct screening and interventions but do not always agree on their approach: The American Academy of Pediatrics (Adamkin \& Committee on Fetus and Newborn, 2011), the Pediatric Endocrine Society (Thornton et al., 2015), and the Academy of Breastfeeding Medicine (Wight, Marinelli, \& Academy of Breastfeeding Medicine, 2014). All guidance documents agree that breastfeeding is important and should continue whether or not the infant is at risk or undergoing treatment for hypoglycemia.

Infants at risk for hypoglycemia include small for gestational age, large for gestational age, preterm or late preterm, infants of diabetic mothers, those who have a suspected infection, who have experienced perinatal stress, who were delivered by cesarean section, who have certain inborn errors of metabolism or endocrine disorders, and who have been exposed to cold stress. Betamimetic drugs, such as Ephedrine (used to treat maternal hypotension from epidural analgesia) or Terbutaline, given just before birth can cause maternal hyperglycemia and aggravate the risk for newborn hypoglycemia (Shields \& Tsay, 2012). Typically, newborns with no risk factors are not screened for hypoglycemia (Wight et al., 2014).

Infants who are asymptomatic and at risk or fail to normalize their blood glucose levels by breastfeeds alone have been treated with bolus formula feedings and, if necessary, with intravenous (IV) glucose infusions, which usually necessities transfer to the neonatal intensive care unit (NICU). In an effort to reduce NICU admissions and minimize the use of infant formula, many hospitals now use a $40 \%$ glucose gel applied to the infant's buccal mucosa as an integral part of their hypoglycemia protocol (Rawat et al., 2016). Glucose gel intervention has resulted in a reduction in the need for IV therapy, decreased formula supplementation, increased exclusive breastfeeding rates, and has been shown to be a costeffective intervention with high patient satisfaction (Rawat et al., 2016; Stewart, Sage, \& Reynolds, 2016).

\section{Implications for Practice}

Interventions for hypoglycemia can include:

- Frequent breastfeedings (10-12 times/24 hours) with documented swallowing to keep a steady supply of calories and glucose available to the infant. Colostrum has been shown to stabilize newborn glucose levels as effectively as formula in the first 4 hours after birth (Tozier, 2013). Tozier (2013) reported a protocol that involved hand expressing colostrum periodically during labor, storing this in feeding syringes, and feeding this colostrum to the infant during the first hour after birth along with feedings at breast and continuous skin-to-skin contact.

Postbirth colostrum expression continued such that the infant received colostrum drops every couple of hours in addition to frequent breastfeeding efforts. This protocol reduced formula supplementation and NICU admissions. A steady supply of colostrum rather than intermittent receipt of colostrum boluses may prevent glucose level swings and help stabilize blood glucose levels better. The approach of a more continuous provision of glucose through hand expressed colostrum, even hourly during the transitional first hours after birth, would be a logical and perhaps more physiological intervention to handle the challenge of hypoglycemia since the infant experiences a steady supply of glucose in utero.

- Supplementation amounts have received much debate. Santoro, Martinez, Ricco, and Jorge (2010) found that during the first 24 hours following birth, breastfed infants ingested $15 \pm 11 \mathrm{~g}$ of colostrum total with a mean weight gain of $1.5 \pm 1.1 \mathrm{~g}$ per breastfeeding over 9-10 feedings. The stomach capacity of newborns has been calculated to be anywhere from 12 to $35 \mathrm{ml}$, with an average of $20 \mathrm{ml}$ (Bergman, 2013). However, just because the capacity exists does not mean that the infant will actually consume that amount at each feeding, especially on the first day of life when the stomach is rather noncompliant. While the stomach has the ability to expand and does so as the infants get older, it is unrealistic to expect a young infant to consume a Thanksgiving dinner at every feeding.

An infant who is spitting up may indicate that the amount of food ingested does not fit into the stomach. Bergman (2013) discusses hourly feeds of the newborn as a mechanism to avoid hypoglycemia and calculates the increasing capacity of the infant's stomach during the first month of life as a linear growth pattern of $7 \mathrm{ml} / \mathrm{kg}$ of body weight. For supplementation volumes, healthy breastfed, term infants could typically consume $2-10 \mathrm{ml} /$ feed during the first 24 hours, $5-15 \mathrm{ml} /$ feed from 24 to 48 hours, $15-30 \mathrm{ml} /$ feed from 48 to 72 hours, and $30-60 \mathrm{ml} /$ feed from 72 to 96 hours (Kellams, Harrel, Omage, Gregory, \& Rosen-Carole, 2017). 
- Avoid cold stress and infant crying and keep the infant skin-to-skin. Glucose levels have been shown to be higher when the infant is placed skin-to-skin rather than separated and placed in a bassinet, NICU admissions are reduced, and overall physiologic stability is enhanced (Chiruvolu et al., 2017).

- Delay the initial bath until 12 hours or later following birth to reduce cold stress and promote immediate skin-to-skin care.

- Diabetic mothers can hand express colostrum during the prenatal period, bringing it to the hospital with them in case the infant requires supplementation (Forster et al., 2017). This assures a ready supply during the first hours postbirth when the potential for hypoglycemia is highest.

\section{Excessive Weight Loss, Dehydration, and Jaundice}

Excessive weight loss, dehydration, and jaundice are a trio of unwelcome and interconnected entities. Neonatal hypernatremia/dehydration is strongly associated with weight loss (Oddie, Craven, Deakin, Westman, \& Scally, 2013), and excessive weight loss is strongly associated with increased bilirubin levels (Hassan \& Zakerihamidi, 2018). Effective breastfeeding plays a major role in reducing the appearance of these unwanted visitors to the breastfeeding family. Hassan and Zakerihamidi (2018) reported that the average serum bilirubin levels in newborns with less than seven breastfeedings per day was higher than those newborns who fed more than seven times per day, that with the increased frequency of feedings, the percentage of weight loss decreased, and that bilirubin levels also decreased with the resulting increase in stooling and decrease in percentage of weight loss.

In order to obtain a more accurate picture of normal weight versus excessive/dangerous weight loss, some researchers and hospital protocols use the 24-hour weight as the reference for weight loss calculation (Deng \& McLaren, 2018; Mulder \& Gardner, 2015). This accounts for the diuresis of excess fluid that results in an inflated birth weight due to maternal intrapartum IV fluids.

Capillary blood ketone levels during the first 48 hours have been used to determine the adequacy of colostrum/breast milk intake during the early hours and days following birth. Bedside ketone measurement may yield rapid information regarding insufficient intake of calories, energy depletion, and the presence of dehydration (Futatani et al., 2017). A more thorough discussion of neonatal weight loss is covered by Genna and Notarangelo in this issue of Clinical Lactation. Many of the same risk factors for neonatal hypoglycemia and delayed onset of lactation also exist for excessive weight loss, dehydration, and jaundice.

\section{Implications for Practice}

- In-hospital and postdischarge feedings plans are imperative to avoid poor breastfeeding outcomes. These feeding plans should be written out and can even be sent to a smartphone to assure that parents understand the infant's feeding needs and have the skills to effectively breastfeed (Appendix B), can recognize yellow and red flags (Appendix C), and have a mechanism to secure immediate help.

- Provision of infant formula to newborns is often the first intervention for excessive weight loss, dehydration, hypoglycemia, and jaundice. Some researchers believe that formula supplementation should automatically begin if a newborn loses $\geq 5 \%$ of his/her birth weight with supplementation of $10 \mathrm{ml}$ of formula to be given following each breastfeeding (Flaherman et al., 2013; Flaherman et al., 2018). However, brief exposure to formula in the hospital can result in disturbed infant's gut microbiota, disrupting the normal bacterial colonization of the infant's gut, and permanently altering the infant's gut microbiome (Forbes et al., 2018).

- Many hospitals use pasteurized donor human milk rather than infant formula should a breastfed infant require supplementation (Lewis et al., 2018). This avoids potential harm to the infant's gut microbiome. If continued supplementation is required postdischarge, parents can purchase donor human milk from a milk bank (Mothers' Milk Bank Northeast, 2018). Insurance reimbursement for banked pasteurized donor human milk is inconsistent, with Medicaid coverage mandated in California, Kansas, Missouri, New York, Texas, Utah, and Washington, DC.

- Breastfeeding dyads with risk factors or impaired breastfeeding in the hospital require a referral to an outpatient source of lactation support such as an IBCLC. Contact should be arranged prior to discharge to avoid delays in securing help which can contribute to an escalating intensity of early problems. 
- Many breastfeeding parents reach out to the internet or other social media platforms when they have questions or to seek help with breastfeeding concerns rather than contacting a healthcare provider or IBCLC. They may also rely on suggestions from friends, relatives, or even strangers. While the internet and social media can provide valuable support, they cannot take the place of expert professional lactation guidance, especially when a potentially serious breastfeeding problem is present.

Because the digital expanse provides a comfort zone for so many parents, the IBCLC or other lactation care provider may wish to utilize social media platforms as an additional mechanism for providing very close monitoring and follow-up of at-risk dyads. Phone contact and in-person visits remain important, but clinicians might also consider platforms such as Twitter, Skype, Facetime, Instagram, and two-way text messaging (Martinez-Brockman, Harari, \& Pérez-Escamilla, 2018), as some parents may seldom seek breastfeeding advice or help in person (Audelo, 2014). These interactive platforms can provide timely and efficient responses to breastfeeding needs.

The first 14 days following delivery are crucial in establishing sufficient milk production and an infant who is breastfeeding successfully. One study showed that a text messaging intervention resulted in a shorter time-to-contact, with some mothers using the text messaging platform either the same day as their baby was born or less than 2 days postpartum (Martinez-Brockman et al., 2018). Such an early opportunity for contact, either initiated by the mother or the clinician, could greatly expedite the identification and resolution of breastfeeding difficulties, particularly those that social media postings identify as problematic.

- Comprehensive discharge teaching (Appendix D) can be accomplished over the course of the hospital stay to avoid overwhelming parents at the last minute.

\section{Conclusions}

Exclusive breastfeeding for the first 6 months remains the accepted public health objective it always has. It is not fraught with danger nor will scare tactics improve the health of our mothers and infants. Comprehensive education and timely, current, and effective interventions and support will help families meet their breastfeeding goals.

\section{References}

Adamkin, D. H., \& Committee on Fetus and Newborn. (2011). Postnatal glucose homeostasis in late-preterm and term infants. Pediatrics, 127(3), 575-579. http://dx.doi.org/10.1542/peds. 2010-3851

Adamkin, D. H., \& Polin, R. (2016). Neonatal hypoglycemia: Is 60 the new 40? The questions remain the same. Journal of Perinatology, 36(1), 10-12. http://dx.doi.org/10.1038/jp.2015. $\underline{125}$

American College of Obstetricians and Gynecologists. (2018a). Letter to Members. Accessed April 1, 2018.

American College of Obstetricians and Gynecologists. (2018b). ACOG Rounds. Retrieved from https://www.magnetmail.net/ actions/email web version.cfm?ep=QDITH zQAwsR6M236W p2YC34MWCuldxNM2BzJ8YBsv0Gd2Avhnq8RS3vZFT8oskBjemrqz9Qzrtq7-jKa4flMLYTWOrunX70MCBgTsom6w ggdJL21O 3CaOOedCi3s4

Arthur, P. G., Kent, J. C., \& Hartmann, P. E. (1994). Metabolites of lactose synthesis in milk from diabetic and nondiabetic women during lactogenesis II. Journal of Pediatric Gastroenterology and Nutrition, 19(1), 100-108. http://dx.doi.org/10.1097/00005176199407000-00017

Audelo, L. (2014). Connecting with today's mothers: Breastfeeding support online. Clinical Lactation, 5(1), 16-19. http://dx.doi. org $/ 10.1891 / 2158-0782.5 .1 .16$

Bergman, N. J. (2013). Neonatal stomach volume and physiology suggest feeding at 1-h intervals. Acta Paediatrica, 102(8), 773-777. http://dx.doi.org/10.1111/apa.12291

Bitman, J., Hamosh, M., Hamosh, P., Lutes, V., Neville, M. C., Seacat, J., \& Wood, D. L. (1989). Milk composition and volume during the onset of lactation in a diabetic mother. The American Journal of Clinical Nutrition, 50(6), 1364-1369. http://dx.doi. org/10.1093/ajcn/50.6.1364

Chiruvolu, A., Miklis, K. K., Stanzo, K. C., Petrey, B., Groves, C. G., McCord, K., \& Tolia, V. N. (2017). Effects of skin-to-skin care on late preterm and term infants at risk for neonatal hypoglycemia. Pediatric Quality and Safety, 2(4), e030.

Deng, X., \& McLaren, M. (2018). Using 24-hour weight as reference for weight loss calculation reduced supplementation and promotes exclusive breastfeeding in infants born by cesarean section. Breastfeeding Medicine, 13(2), 128-134. http://dx.doi. org $/ 10.1089 / \mathrm{bfm} .2017 .0124$

Dewey, K. G., Nommsen-Rivers, L. A., Heinig, M. J., \& Cohen, R. J. (2003). Risk factors for suboptimal infant breastfeeding behavior, delayed onset of lactation, and excess neonatal weight loss. Pediatrics, 112(3 Pt 1), 607-619. http://dx.doi.org/10.1542/ peds.112.3.607

Donath, S. M., \& Amir, L. H. (2008). Maternal obesity and initiation and duration of breastfeeding: Data from the longitudinal study of Australian children. Maternal and Child Nutrition, 4(3), 163-170. http://dx.doi.org/10.1111/j.1740-8709. 2008.00134.x

Flaherman, V. J., Aby, J., Burgos, A. E., Lee, K. A., Cabana, M. D., \& Newman, T. B. (2013). Effect of early limited formula on duration and exclusivity of breastfeeding in at-risk infants: An 
RCT. Pediatrics, 131(6), 1059-1065. http://dx.doi.org/10.1542/ peds.2012-2809

Flaherman, V. J., Narayan, N. R., Hartigan-O'Connor, D., Cabana, M. D., McCulloch, C. E., \& Paul, I. M. (2018). The effect of early limited formula on breastfeeding, readmission, and intestinal microbiota: A randomized clinical trial. The Journal of Pediatrics, 196, 84-90. http://dx.doi.org/10.1016/i.jpeds.2017.12.073

Forbes, J. D., Azad, M. B., Vehling, L., Tun, H. M., Konya, T. B., Guttman, D. S, Kozyrskyj, A. L., . . Canadian Healthy Infant Longitudinal Development (CHILD) Study Investigators. (2018). Association of exposure to formula in the hospital and subsequent infant feeding practices with gut microbiota and risk of overweight in the first year of life. JAMA Pediatrics, 172(7), e181161. http://dx.doi.org/10.1001/jamapediatrics.2018.1161

Forster, D. A., Moorhead, A. M., Jacobs, S. E., Davis, P. G., Walker, S. P., McEgan, K. M., ... Amir, L. H. (2017). Advising women with diabetes in pregnancy to express breastmilk in late pregnancy (Diabetes and Antenatal Milk Expressing [DAME]): a multicentre, unblinded, randomised controlled trial. The Lancet, 389(10085), 2204-2213. http://dx.doi.org/10.1016/S01406736(17)31373-9

Futatani, T., Shimao, A., Ina, S., Higashiyama, H., Fujita, S., Ueno, K., . . . Hatasaki, K. (2017). Capillary blood ketone levels as an indicator of inadequate breast milk intake in the early neonatal period. The Journal of Pediatrics, 191, 76-81. http://dx.doi.org/ 10.1016/i.jpeds.2017.08.080

Hartmann, P., \& Cregan, M. (2001). Lactogenesis and the effects of insulin-dependent diabetes mellitus and prematurity. The Journal of Nutrition, 131(11), 3016S-3020. http://dx.doi.org/10.1093/ in/131.11.3016S

Hassan, B., \& Zakerihamidi, M. (2018). The correlation between frequency and duration of breastfeeding and the severity of neonatal hyperbilirubinemia. The Journal of Maternal-Fetal and Neonatal Medicine, 31(4), 457-463. http://dx.doi.org/10.1080/ $\underline{14767058.2017 .1287897}$

Hilson, J. A., Rasmussen, K. M., \& Kjolhede, C. L. (2004). High prepregnant body mass index is associated with poor lactation outcomes among white, rural women independent of psychosocial and demographic correlates. Journal of Human Lactation, 20(1), 18-29. http://dx.doi.org/10.1177/ $\underline{0890334403261345}$

Kair, L. R., \& Colaizy, T. T. (2016). Obese mothers have lower odds of experiencing pro-breastfeeding hospital practices than mothers of normal weight: CDC Pregnancy Risk Assessment Monitoring System (PRAMS), 2004-2008. Maternal and Child Health Journal, 20(3), 593-601. http://dx.doi.org/10.1007/ $\underline{\text { s10995-015-1858-z }}$

Kellams, A., Harrel, C., Omage, S., Gregory, C., Rosen-Carole, C., \& the Academy of Breastfeeding Medicine. (2017). ABM clinical protocol \#3: Supplementary feedings in the healthy term breastfed neonate, revised 2017. Breastfeeding Medicine, 12(4), 188-198. http://dx.doi.org/10.1089/bfm.2017.29038.ajk

Kendall-Tackett, K. (2018). Inflammatory rhetoric aside. is there anything to learn from Fed is Best? Clinical Lactation, 9(2), 6-7.

Lewis, S. C., McMahon, M., Combs, G., Smith, K., SeguraHarrison, M., \& Philipp, B. L. (2018). The nuts and bolts of implementing a pasteurized donor human milk program on a mother baby unit. Journal of Human Lactation, 34(1), 116-119. http://dx.doi.org/10.1177/0890334417740346

Martinez-Brockman, J. L., Harari, N., \& Pérez-Escamilla, R. (2018). Lactation advice through texting can help: An analysis of intensity of engagement via two-way text messaging. Journal of Health Communication, 23(1), 40-51. http://dx.doi.org/10. $\underline{1080 / 10810730.2017 .1401686}$

Miyake, A., Tahara, M., Koike, K., \& Tanizawa, O. (1989). Decrease in neonatal suckled milk volume in diabetic women. European Journal of Obstetrics and Gynecology and Reproductive Biology, 33(1), 49-53. http://dx.doi.org/10.1016/0028-2243(89)90077-4

Mothers' Milk Bank Northeast. (2018). Receive milk. Retrieved from https://milkbankne.org/receive/

Mulder, P. J., \& Gardner, S. E. (2015). The healthy newborn hydration model: A new model for understanding newborn hydration immediately after birth. Biological Research for Nursing, 17(1), 94-99. http://dx.doi.org/10.1177/1099800414529362

Murtaugh, M. A., Ferris, A. M., Capacchione, C. M., \& Reece, E. A. (1998). Energy intake and glycemia in lactating women with type 1 diabetes. Journal of the American Dietetic Association, 98(6), 642-648. http://dx.doi.org/10.1016/S0002-8223(98)00147-3

Oddie, S. J., Craven, V., Deakin, K., Westman, J., \& Scally, A. (2013). Severe neonatal hypernatraemia: A population based study. Archives of Disease in Childhood - Fetal and Neonatal Edition, 98(5), F384-F387. http://dx.doi.org/10.1136/archdischild$\underline{2012-302908}$

Ostrom, K. M., \& Ferris, A. M. (1993). Prolactin concentrations in serum and milk of mothers with and without insulin-dependent diabetes mellitus. The American Journal of Clinical Nutrition, 58(1), 49-53. http://dx.doi.org/10.1093/ajcn/58.1.49

Preusting, I., Brumley, J., Odibo, L., Spatz, D. L., \& Louis, J. M. (2017). Obesity as a predictor of delayed lactogenesis II. Journal of Human Lactation, 33(4), 684-691. http://dx.doi.org/10.1177/ $\underline{0890334417727716}$

Rasmussen, K. M., \& Kjolhede, C. L. (2004). Prepregnant overweight and obesity diminish the prolactin response to suckling in the first week postpartum. Pediatrics, 113(5), e465-e471. http://dx.doi.org/10.1542/peds.113.5.e465

Rasmussen, K. M., Lee, V. E., Ledkovsky, T. B., \& Kjolhede, C. L. (2006). A description of lactation counseling practices that are used with obese mothers. Journal of Human Lactation, 22(3), 322-327. http://dx.doi.org/10.1177/0890334406290177

Rawat, M., Chandrasekharan, P., Turkovich, S., Barclay, N., Perry, K., Schroeder, E., . . . Lakshminrusimha, S. (2016). Oral dextrose gel reduces the need for intravenous dextrose therapy in neonatal hypoglycemia. Biomedicine Hub, 1(3), 448511. http://dx.doi.org/10.1159/000448511

Santoro, W., Martinez, F. E., Ricco, R. G., \& Jorge, S. M. (2010). Colostrum ingested during the first day of life by exclusively breastfed healthy newborn infants. The Journal of Pediatrics, 156(1), 29-32. http://dx.doi.org/10.1016/i.jpeds.2009.07.009

Seske, L. M., Merhar, S. L., \& Haberman, B. E. (2015). Late-onset hypoglycemia in term newborns with poor breastfeeding. Hospital Pediatrics, 5(9), 501-504. http://dx.doi.org/10.1542/ hpeds.2015-0086 
Shields, L., \& Tsay, G. S. (Eds.). (2012). California Diabetes and Pregnancy Program Sweet Success Guidelines for Care. Developed with California Department of Public Health; Maternal, Child and Adolescent Health Division; revised edition, July, 2012. http:// perinatology.com/Reference/CDAPP\%20SS\%20Guidelines\% 202012.pdf

Stewart, C. E., Sage, E. L. M., \& Reynolds, P. (2016). Supporting 'baby friendly': A quality improvement initiative for the management of transitional neonatal hypoglycaemia. Archives of Disease in Childhood - Fetal and Neonatal Edition, 101(4), F344F347. http://dx.doi.org/10.1136/archdischild-2015-308950

Thornton, P. S., Stanley, C. A., De Leon, D. D., Harris, D., Haymond, M. W., Hussain, K, Wolfsdorf, J.I., . . Pediatric Endocrine Society. (2015). Recommendations from the Pediatric Endocrine Society for evaluation and management of persistent hypoglycemia in neonates, infants, and children. The Journal of
Pediatrics, 167(2), 238-245. http://dx.doi.org/10.1016/i.jpeds. $\underline{2015.03 .057}$

Tozier, P. K. (2013). Colostrum vs formula supplementation for glucose stabilization in newborns of diabetic mothers. Journal of Obstetric, Gynecologic and Neonatal Nursing, 42(6), 619-628. http://dx.doi.org/10.1111/1552-6909.12260

Vice, F. L., Heinz, J. M., Giuriati, G., Hood, M., \& Bosma, J. F. (1990). Cervical auscultation of suckle feeding in newborn infants. Developmental Medicine and Child Neurology, 32(9), 760768. http://dx.doi.org/10.1111/j.1469-8749.1990.tb08479.x

Wight, N., Marinelli, K. A., \& Academy of Breastfeeding Medicine. (2014). ABM clinical protocol \#1: Guidelines for blood glucose monitoring and treatment of hypoglycemia in term and latepreterm neonates, revised 2014. Breastfeeding Medicine, 9(4), 173-179. http://dx.doi.org/10.1089/bfm.2014.9986

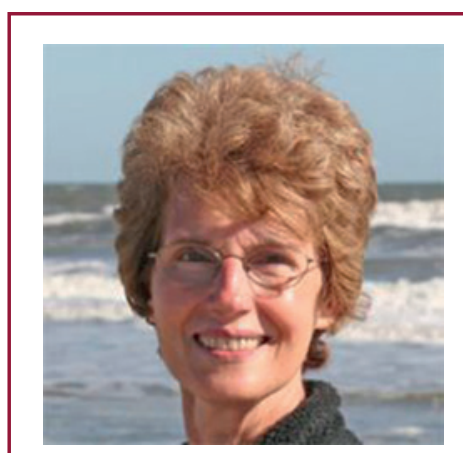

Marsha Walker, RN, IBCLC, RLC, is a registered nurse and international board certified lactation consultant. She is the executive director of the National Alliance for Breastfeeding Advocacy (NABA) and a member of the board of directors of the Massachusetts Lactation Consultant Association. She is USLCA's representative to the USDA's Breastfeeding Promotion Consortium and NABA's representative to the U.S. Breastfeeding Committee. She is the author of Breastfeeding Management for the Clinician: Using the Evidence, 4th edition, an advocate for breastfeeding at the state and federal levels, and a frequent speaker on lactation issues.

\section{Best States to Work Index, From Oxfam}

Oxfam has released a research report titled, "The Best States to Work Index: A Guide to Labor Policy in US States": https://policy-practice.oxfamamerica.org/static/media/files/Best States to Work Index.pdf In an effort to understand the differences between states and to rank them, Oxfam constructed an index of labor and employment policies of all 50 states and Washington, DC. The result is the Best States to Work Index. Using this tool, legislators, labor advocates, and residents may see how the score of their state compares overall and in their region, identify labor policy priorities, and recognize underperforming states. This report also includes an in-depth analysis of state trends in three areas -wage, worker protection, and the right to organize. It attempts to identify low-hanging fruit, where efforts for improvement would likely yield quick results, through a strategic analysis of trends based on a comparison of the three dimensions, as well as trends in individual policies. Oxfam specifically recommends providing workplace protections for pregnant and breastfeeding workers.

Source: USBC. 
Appendix A.

\section{BOMDILW}

Skin-to-skin contact during the first hour post birth and liberally throughout the hospital stay

- Prevents chilling and crying (both of which lower blood glucose levels)

* Alerts you to subtle infant feeding cues

Feed your baby frequently to assure a steady supply of calories and glucose.

+ Within 1 hour after birth

* Once every hour for the next 3 to 4 hours

+ Every 2 to 3 hours until 12 hours of age

+ At least 8-12 times each 24 hours during your hospital stay

Move baby to breast when baby shows feeding behavioral readiness cues.

These cues are especially important to use if your baby is sleepy.

+ Sucking movements of the mouth and tongue

- Rapid eye movements under the eyelids

* Hand-to-mouth movements

- Body movements

+ Small sounds

Make sure you know how to tell when your baby is SWALLOWING. This is very important to assure adequate intake both during vour hospital stav and once. vou are home. Some babies latch and suck but do not feed efficiently enough to swallow.

+ Baby's jaw drops and holds for a second

+ You hear a "ca" sound or a puff of air from the nose

+ You feel a drawing action on the areola and see it move towards your baby's mouth

You hear the baby swallow

* You feel the swallow when you place a finger on the baby's throat

+ Your nurse hears the swallow when a stethoscope is placed on the baby's throat

* If you are uncertain if the baby is swallowing, ask that a pre- and post-feed weight be done to assure that the baby is receiving nourishment

Use alternate massage if your baby doesn't swallow after every 1 to 3 sucks.

+ Massage and squeeze the breast each time she stops between sucks.

+ This helps get more colostrum into her and keeps her sucking longer.

If your baby does not latch or swallow when at the breast or falls asleep quickly then hand express colostrum into a teaspoon or use a syringe, dropper, or small medicine cup to feed this expressed colostrum to your baby.

+ Feed your baby 2-10 mLfeed (1-2 teaspoons) during the first 24 hours, 5-15 mL/feed from 24-48 hours (1-3 teaspoons), 15-30 mL/feed from 48-72 hours 3 teaspoons to 1 ounce or so), and 30-60 mLlfeed (1-2 ounces) from 72-96 hours

* At each feeding attempt it is important that baby receives either your colostrum or banked donor human milk if not enough colostrum is available. Avoid skipped feedings

You can feed your baby drops of colostrum with a feeding syringe between feeding's to keep a steady supply of calories and glucose getting into baby. 
Appendix B.

\section{MY HOSPITAL DISCHARGE CHECKLIST}

- I can position my baby correctly on both breasts

It does not hurt once the baby starts sucking

a The baby can latch to each breast

a I can tell when the baby is swallowing milk

a I know how many times in $\mathbf{2 4}$ hours to feed the baby

a I know when my milk should come in \& who to call if it doesn't by day 3

a I know how long to feed the baby on each side

a I know that feedings lasting longer than 30 minutes is a sign of baby not feeding well

] I know that if baby does not feed well from the breast I should hand express colostrum and/or pump milk and feed the baby as much of that milk

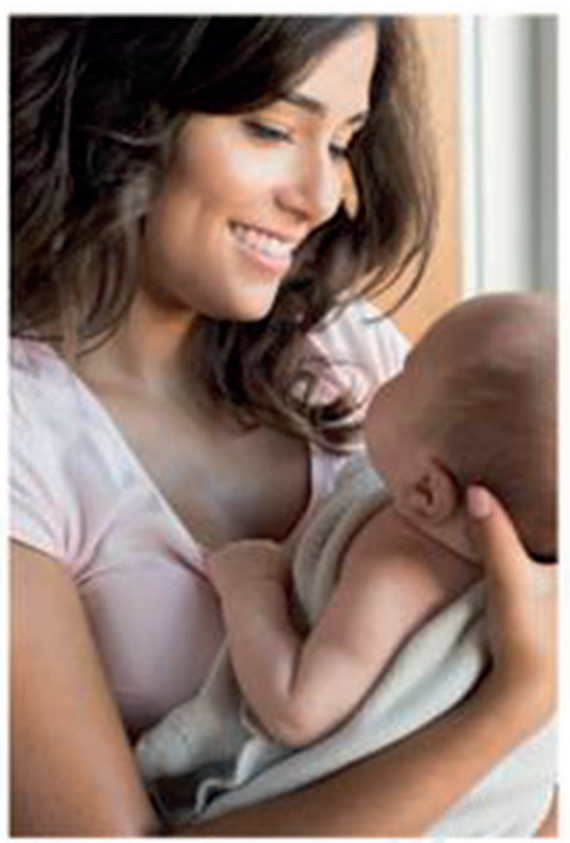
as he will take

a I know when it is time to feed my baby

a I know the five feeding cues to use if my baby is sleepy

- I know how many diapers baby should have each day

a I know how to tell if a disposable diaper is wet

] I know how much weight baby should gain weekly

] I know that artificial nipples and pacifiers can confuse my baby and have been shown other ways to feed him if necessary

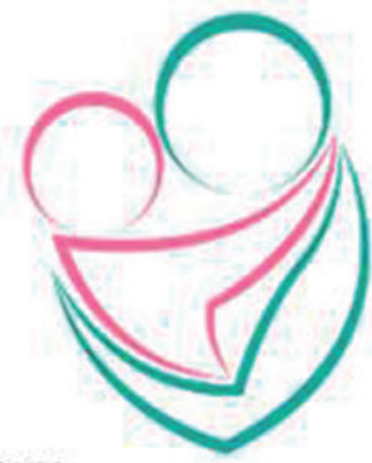

I I know I should hand express and pump milk if baby does not feed well

I I know that persistent, inconsolable crying may be a sign of underfeeding and will feed baby expressed colostrum or pumped milk

I know how to tell if my baby is jaundiced

ב Someone will visit me a day or two after I get home, or....

I will see my pediatrician or family doctor in one to two days

a I know when and who to call for help with nursing if I have any concerns 


\section{LOOK! LISTEN! ACT!}

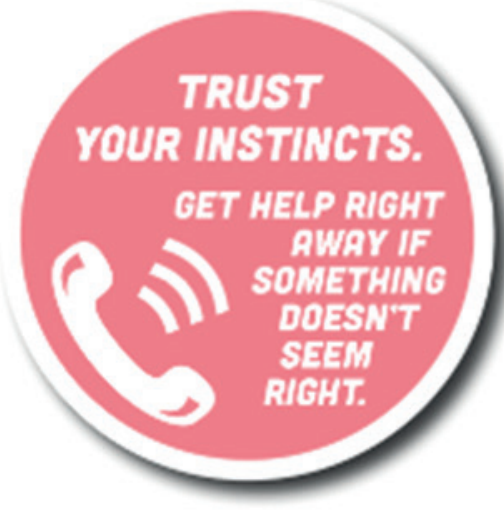

CALL YOUR HEALTHCARE

PROVIDER RIGHT AWAY IF;

- Baby does not have a wet diaper for longer than 6 hours

- Urine is dark and smells strong

- Baby is lethargic, limp or docile

- Baby has dry mouth and no tears

- Baby is irritable

- Baby has inconsolable crying

- Baby has a sunken fontanel

- Baby is feverish

- Baby has yellow skin

- Skin when pinched remains tented up

\section{WHERE TO FIND HELP}

To locate a lactation consultant: www.USLCA.org

To find a variety of breastfeeding support services: www.zipmilk.org

App for $24 / 7$ breastfeeding support: www.pacify.com

Office on Women's Health Helpline: 800-994-9662

My local number for help:

WC Offices - La Leche League State Breastfeeding Coalitions - Baby Cafes Local breastfeeding support organizations

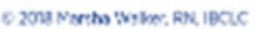

\section{BREASTFEEDING PROBLEMS CAN HAPPEN!} Know when and where to seek help!

Most breastfeeding mothers find that the early days of nursing a baby is a time of exploring and learning what works best for both mom and baby. With basic instruction and support from your healthcare providers, breastfecding usually gets off to a good start. However, sometimes mother- nature throws you a curve ball and problems crop up that need attention right away. Learning

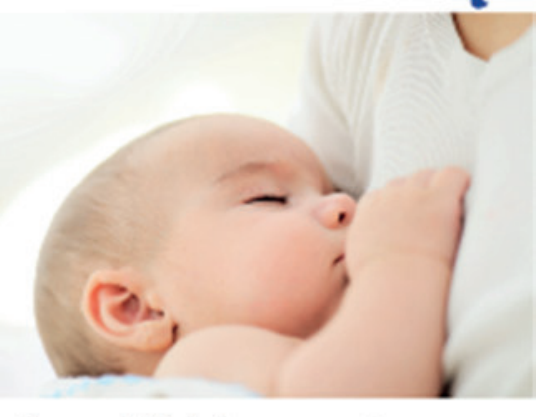
to recognize these problems and act on them quickly helps you meet your breastleeding goals and enjoy a satislying breasteeding relationship.

\section{CMOSESFOH GOW SERM}

Make sure you are working with an IBCLC (International Board Certified Lactation Consuitant) and contact your IBCLC or other knowledgeable Inciation care provider immediately if: you

- Are a first time mother

- Had a cesarean delivery

- Have a history of low milk supply

- Are diabetic

- Are obese

- Are hypothyroid

- Have polycystic ovary syndrome

- Have tubular or asymmetric breasts

- Took prenatal SSRI medication

- Have sore nipples

- 'Ihink you don't have enough milk

\section{Your Baby}

- Is preterm or late preterm

- Small or large for gestational age

- Had vacuum extraction

- Lost more than $7 \%$ of birth weight

- Cannot be heard or seen to swallow colostrum or milk

- Is a twin or triplet

- Does not latch to the breast

- Has uric acid crystals after day 2

- Is jaundiced (yellow skin or whites of the eyes)

- Cries all the time/never satisfied after feedings

- Takes more than 30 minutes to feed

- Is extremely sleepy

\section{What I need to know}

Feed your baby 8-10 times each 24 hours during the early days.

Put a sleepy baby to breast when you see feeding cues...

- Rapid eye movements under eyelids . Sucking movements of mouth

- Hand-to-mouth movements - Body movements

- Small sounds

* Massage \& compress the breast when baby pauses between sucks.

* Have your lactation consultant/nurse verify that baby is swallowing.

* Make sure you know when baby is swallowing.

* If baby does not latch or nurse well, express your colostrumimilk and feed your baby

2-10 mL/feed ( $1-2$ teaspoons) during the first 24 hours, $3-15 \mathrm{~mL}$ feed from $24-48$

hours ( $1-3$ teaspoons), $15.30 \mathrm{~mL} / \mathrm{f}$ feed from 48.72 hours ( 3 teaspoons to 1 ounce or

so), and $30-60 \mathrm{~mL}$ : feed (1-2 ounces) from $72-96$ hours

* Consult your healthcare provider and $\mathrm{LC}$ if more supplettentation is needed. 


\section{Appendix D.}

\section{Postpartum Discharge Education Checklist}

\section{Nurse or lactation consultant has verified that:}

$\checkmark$ Baby is positioned and latched correctly to both breasts

$\checkmark$ Baby is swallowing while feeding

$\checkmark$ Mother verbalizes that she knows when the baby is swallowing

$\checkmark$ If swallowing is in doubt then pre-and post-feed weights have been done to determine intake

$\checkmark$ Supplementation if needed has been discussed including hand expression and the use of donor human milk

$\checkmark$ Mother knows when and how many times per 24 hours to feed the baby

$\checkmark$ Mother has been taught 5 behavioral feeding cues if baby is sleepy

- Rapid eye movements under eyelids

- Sucking movements of mouth and tongue

- Hand-to-mouth movements

- Body movements

- Small sounds

$\checkmark$ Mother knows that feedings lasting longer than 30 minutes indicate feeding difficulties and will call lactation consultant immediately

$\checkmark$ Mother knows that persistent, inconsolable crying may indicate underfeeding and will call lactation consultant/healthcare provider immediately

$\checkmark$ Mothers who are diabetic, obese, have a history of endocrine problems may have delayed lactogenesis II and have been taught to feed the baby 10-12 times each 24 hours until onset of copious milk production

$\checkmark$ Mother has been taught hand expression and will hand express colostrum or pump breastmilk if baby cannot latch or does not swallow milk during breastfeedings. This will be fed to baby by spoon, dropper, cup during each feeding attempt

$\checkmark$ If mother is in doubt whether or not baby has swallowed colostrum/milk she should hand express or pump and feed the baby

$\checkmark$ Mother has name and contact information for community lactation consultant or other lactation support person

$\checkmark$ Mother has or will secure a breast pump if necessary

$\checkmark$ Mother has appointment with healthcare provider at 2 days post discharge or earlier if necessary

$\checkmark$ Mother has been taught signs of jaundice and to contact her healthcare provider if these are seen

- Yellowing of the sclera

- Yellowing of skin

- Yellow color is seen in skin after pressing gently on baby's nose

- Mother should call physician immediately if baby's skin becomes more yellow, especially on the abdomen, arms or legs or baby becomes listless or is difficult to awaken or has high pitched cries

Mother has been taught signs of dehydration and to contact her healthcare provider immediately

- Mother has been taught diaper outputs and that no wet diaper for longer than 6 hours indicates a call to healthcare provider, as does uric acid crystals after day 2, strong smelling or dark colored urine, or failure to see transitional/yellow stools.

- Other signs indicating a call to the healthcare provider include excessive sleepiness, irritability, less elasticity in the skin, decrease or absence of tears, dry mouth and lips 